NDO with oxygen or hydrogen peroxide, as proposed by Wolfe and Lipscomb $(17,18)$.

The unique feature of NDO that sets it apart from $\mathrm{P} 450$ is its ability to catalyze stereospecific addition of dioxygen to aromatic compounds, resulting in the formation of cis-dihydrodiols $(12,24)$. For cytochrome P450 and MMO, transient high iron oxidation states are required for the necessary oxygen cleavage (25); this is not the case for NDO, where both atoms of dioxygen react in concert with the substrate. The four electrons required for catalysis are one each from the active-site ferrous iron and the Rieske center, and two from the double bond of the substrate. An important difference in the geometry of the active sites of cytochrome P450 and NDO is that the active-site iron is highly accessible in NDO, with a large part of its surface available for the binding of both oxygen atoms to iron. The favorable position of the substrate and $\mathrm{Asn}^{201}$ further favors side-on binding. It is possible that for cytochrome $\mathrm{P} 450$ and other heme proteins, the presence of the planar heme group reduces the accessible surface of iron, resulting in an end-on binding of oxygen.

We have directly observed a side-on dioxygen species bound to iron. Computational studies (26) have predicted the existence of this species, and there are spectroscopic data consistent with side-on binding $(27,28)$. We suggest that side-on binding of oxygen as a starting point for oxygen activation by NDO has direct implications for the mechanism of dihydroxylation of NDO in terms of the stereo- and regiospecific reactions catalyzed by the enzyme (29). The results presented here provide a new basis for further computational and structural investigations of oxygen activation by biological systems and may facilitate the design of chemical catalysts capable of cis-dihydroxylation.

\section{References and Notes}

1. I. Schlichting et al., Science 287, 1615 (2000).

2. T. L. Poulos, Curr. Opin. Struct. Biol. 5, 767 (1995).

3. B. C. Finzel, I. C. Gunsalus, G. C. Wagner, J. Kraut, J. Biol. Chem. 260, 6122 (1985).

4. T. L. Poulos, B. C. Finzel, A. J. Howard, Biochemistry 25, 5314 (1986)

5. T. L. Poulos, R. Raag, FASEB J. 6, 674 (1992)

6. H. Y. Li, S. Narasimhulu, L. M. Havran, J. D. Winkler, T. L. Poulos, J. Am. Chem. Soc. 117, 6297 (1995).

7. B. J. Wallar, J. D. Lipscomb, Chem. Rev. 96, 2625 (1996).

8. A. C. Rosenzweig, C. A. Frederick, S. J. Lippard, P. Nordlund, Nature 366, 537 (1993).

9. A. C. Rosenzweig, P. Nordlund, P. M. Takahara, C. A. Frederick, S. J. Lippard, Chem. Biol. 2, 409 (1995).

10. J. D. Lipscomb, Annu. Rev. Microbiol. 48, 371 (1994).

11. D. T. Gibson, R. E. Parales, Curr. Opin. Biotechnol. 11, 236 (2000).

12. T. Hudlicky, D. Gonzalez, D. T. Gibson, Aldrichim. Acta 32, 35 (1999)

13. B. Kauppi et al., Structure 6, 571 (1998)

14. A. M. Jeffrey et al., Biochemistry 14, 575 (1975)

15. B. E. Haigler, D. T. Gibson, J. Bacteriol. 172, 457 (1990).

16. J. Bacteriol. 172, 465 (1990)

17. M. D. Wolfe, J. V. Parales, D. T. Gibson, J. D. Lipscomb, J. Biol. Chem. 276, 1945 (2001).

18. M. D. Wolfe, J. D. Lipscomb, J. Biol. Chem. 278, 829 (2003).
19. M. D. Wolfe et al., Biochemistry 41, 9611 (2002).

20. K. Chen, L. Que, Angew. Chem. Int. Ed. 38, 2227 (1999).

21. K. Chen, M. Costas, J. Kim, A. K. Tipton, L. Que Jr. J. Am. Chem. Soc. 124, 3026 (2002)

22. E. Carredano et al., J. Mol. Biol. 296, 701 (2000).

23. See supporting data on Science Online.

24. D. R. Boyd, G. N. Sheldrake, Nat. Prod. Rep. 15, 309 (1998)

25. D. L. Harris, Curr. Opin. Chem. Biol. 5, 724 (2001).

26. S. Ahmad et al., Inorg. Chem. 27, 2230 (1988)

27. F. Neese, E. I. Solomon, J. Am. Chem. Soc. 120, 12829 (1998).

28. F. Mather et al., J. Am. Chem. Soc. 124, 4966 (2002).

29. S. M. Resnick, K. Lee, D. T. Gibson, J. Ind. Microbiol. 17, 438 (1996)

30. Z. Otwinowski, Data Collection and Processing (Daresburry Laboratories, Warrington, UK, 1993), pP. $56-62$.

31. J. W. Pflugrath, Acta Crystallogr. D55, 1718 (1999).

32. We thank J. Hajdu and his group at Uppsala for help in carrying out several experiments; $M$. Niebergall for helpful tips; several synchrotron stations and beam lines for help with data collection (European Synchrotron Radiation Facility, Grenoble, Max Lab, Lund, and Industrial Macromolecular Crystallography Association-Collaborative Access Team, Advanced Photon Source); and J. Lipscomb for helpful discussions. Supported by the Swedish Research Council for Environment, Agricultural Science, and Spatial Planning (H.E. and S.R.), the Swedish Research Council (H.E.), and U.S. Public Health Service grants GM62904 (S.R.) and GM29909 (D.T.G.)

\section{Supporting Online Material}

www.sciencemag.org/cgi/content/full/299/5609/1039/ DC1

Materials and Methods

References

Figs. $\mathrm{S} 1$ to $\mathrm{S} 4$

3 September 2002; accepted 19 December 2002

\title{
Carbon Nanotube Flow Sensors
}

\author{
Shankar Ghosh, ${ }^{1}$ A. K. Sood, ${ }^{1 *}$ N. Kumar ${ }^{2}$
}

\begin{abstract}
We report that the flow of a liquid on single-walled carbon nanotube bundles induces a voltage in the sample along the direction of the flow. The voltage that was produced fit a logarithmic velocity dependence over nearly six decades of velocity. The magnitude of the voltage depended sensitively on the ionic conductivity and on the polar nature of the liquid. Our measurements suggest that the dominant mechanism responsible for this highly nonlinear response involves a direct forcing of the free charge carriers in the nanotubes by the fluctuating Coulombic field of the liquid flowing past the nanotubes. We propose an explanation based on pulsating asymmetric ratchets. Our work highlights the device potential for nanotubes as sensitive flow sensors and for energy conversion.
\end{abstract}

Carbon nanotubes are currently being studied in an effort to understand their novel structural, electronic, and mechanical properties and to explore their immense potential for many applications in nanoelectronics (1), and as actuators (2) and sensors (3). A metallic single-walled carbon nanotube (SWNT), which is a low-dimensional system, has electrons that form a strongly correlated liquid, called a Luttinger liquid (4). This liquid shows a separation of spin and charge and demonstrates the associated characteristic power-law dependence of the nanotube resistance on the bias voltage and temperature. Král and Shapiro (5) also showed theoretically the generation of an electric current in a metallic carbon nanotube immersed in a flowing liquid. One mechanism they proposed for the generation of the electric current and voltage is the transfer of momentum from the flowing liquid molecules to the acoustic phonons in the nanotube as the phonon quasi-momentum, which in turn drags free charge carriers in the nanotube. Another

1Department of Physics, Indian Institute of Science, Bangalore 560 012, India. ${ }^{2}$ Raman Research Institute, C.V. Raman Avenue, Bangalore 560 080, India.

*To whom correspondence should be addressed. Email: asood@physics.iisc.ernet.in mechanism noted by these authors involves a direct scattering of the free carriers from the fluctuating Coulombic fields of the ions or polar molecules in the flowing liquid. They argued, however, that the latter mechanism creates a current that is five orders of magnitude smaller than the current that results from the phonon-induced electron drag.

We report the experimental observation of the voltage generated by the flow of a polar liquid over SWNT bundles. It was observed that the induced voltage tends to saturate at flow velocities as low as $10^{-5} \mathrm{~m} / \mathrm{s}$. The observed data can be fitted empirically to a logarithmic dependence of the voltage on the flow velocity, in sharp contrast to the linear dependence predicted by Král and Shapiro (5). The magnitude of the voltage induced along the nanotube by the flow depends significantly on the ionic strength of the flowing liquid, suggesting that the mechanism involving fluctuating Coulombic fields dominates that of the phonon-mediated electron drag. This has led us to propose a mechanism for the observed flow-induced voltage based on a pulsating asymmetrical thermal ratchet model. This mechanism involves the fluctuating Coulombic potentials that result from the ionic liquid in flow, where the asymmetry is provided by the velocity gradient (shear) at the liquid-solid interface. 
The SWNT bundles were prepared by the electric-arc method, followed by purification processes (6). These nanotube bundles, which have an average tube diameter of 1.5 $\mathrm{nm}$, were densely packed between two metal electrodes. The dimensions of the sensor were $1 \times 10^{-3} \mathrm{~m}$ along the flow, $2 \times 10^{-4}$ $\mathrm{m}$ thick, and $2 \times 10^{-3} \mathrm{~m}$ wide. The resistivity of the material was about $0.02 \mathrm{ohm} \cdot \mathrm{m}$, and the sensor had a linear current-voltage characteristic (up to $20 \mathrm{mV}$ ). To avoid any turbulent flow that may have been caused by the expansion of the flow at the inlet of the flow chamber, we placed the nanotube sensor at the center of a glass tube with an inner diameter of $\sim 0.03 \mathrm{~m}$ and a length of $\sim 0.9 \mathrm{~m}$. The schematic of the experimental setup is shown in Fig. 1. The velocity $u$ of the liquid flowing past the sample was measured from the bulk flow rate, which was varied by adjusting the height of the reservoir and the opening of the valve. The liquid enters the flow chamber against gravity such that the formation of air pockets is avoided. The flow at the center of the cell is expected to be laminar because the Reynolds number $(\mathrm{Re})(\sim 300$ for a velocity of $10^{-2} \mathrm{~m} / \mathrm{s}$ ) was much less than the critical value of 2000 for the onset of turbulent flow in a pipe. The measurements shown here were taken after the transients had subsided.

When the sample was immersed in water at rest, a voltage $(\sim 1 \mathrm{mV})$ developed along the sample as an electrochemical potential difference at the interface of the SWNT bundle with the metal electrodes. This offset voltage was subtracted from the voltage measured at finite velocities of the liquid to obtain the flow-induced voltage. The voltage developed only along the flow direction; no voltage was generated in the direction perpendicular to the flow (i.e., along the width).

Figure 1 shows the observed dependence of the flow-induced voltage on the flow velocity of water. Even at the very low flow velocity of $5 \times 10^{-6} \mathrm{~m} / \mathrm{s}$, a voltage of 0.65 $\mathrm{mV}$ is generated. The solid line in Fig. 1 is a fit to the empirical relation $V=\alpha \log (u \beta+$ 1 ), where $\alpha$ and $\beta$ are parameters, with $\alpha=$ $0.6 \mathrm{mV}$ and $\beta=6.5 \times 10^{6} \mathrm{~s} / \mathrm{m}$ for best fit. The induced voltage for a fluid flow velocity of $1.8 \times 10^{-3} \mathrm{~m} / \mathrm{s}$ was $2.67 \mathrm{mV}$ for a sample length of $1 \times 10^{-3} \mathrm{~m}$ and was $1.64 \mathrm{mV}$ for a sample length of $0.5 \times 10^{-3} \mathrm{~m}$, suggesting a one-dimensional bulk property rather than any local tip effects. Figure 2 compares the voltage developed along the nanotubes as a function of the flow velocity for a series of fluid mixtures. A notable effect is the strong dependence of the flow-induced voltage on the ionic strength of the flowing liquid. It can be seen that for $u=10^{-4} \mathrm{~m} / \mathrm{s}$, the voltage for 1.2 $\mathrm{M} \mathrm{HCl}$ is about five times that for water.

We compare our experimental results with those expected from the calculations of Král and Shapiro (5). They considered metallic nanotubes that were densely packed in two layers separated by a layer of water of thickness $d(\sim 10 \mathrm{~nm})$. In their mechanism, the force exerted by the liquid with a flow velocity $u$ on the surface of the nanotubes is $F=(\eta u / d) \times$ interface periphery $X$ interface length. This force results in the steady-state quasi-momentum $(p)$ transfer to the phonon bath, with $p \sim F \tau$, where $\eta$ is the shear viscosity of the liquid and $\tau$ is the phonon umklapp time in the nanotubes. Using this value of $p$, we calculated the steady-state electron distribution from the Boltzmann equation containing the electron-phonon scattering term, which, in turn, gave the hot phonon-induced electronic current $\left(J_{e-p}\right)$. In this model, the calculated current $J_{e-p}$ is directly proportional to the flow velocity $u$ and to the liquid viscosity $\eta$. However, as is shown in Fig. 1, the experimentally observed voltage induced by the flow is nearly logarithmic in the flow velocity $u$. To check for the dependence of the voltage on $\eta$, we repeated the experiments using water-glycerol mixtures. The mixtures used were water: glycerol $=88: 12(\eta=113 \mathrm{mPa} \mathrm{s})$ and water: glycerol $=75: 25(\eta=234 \mathrm{mPa} \mathrm{s})$. These mixtures have viscosities about two orders of magnitude higher than that of water $(\eta=0.9$ $\mathrm{mPa}$ s). We observed that the flow-induced
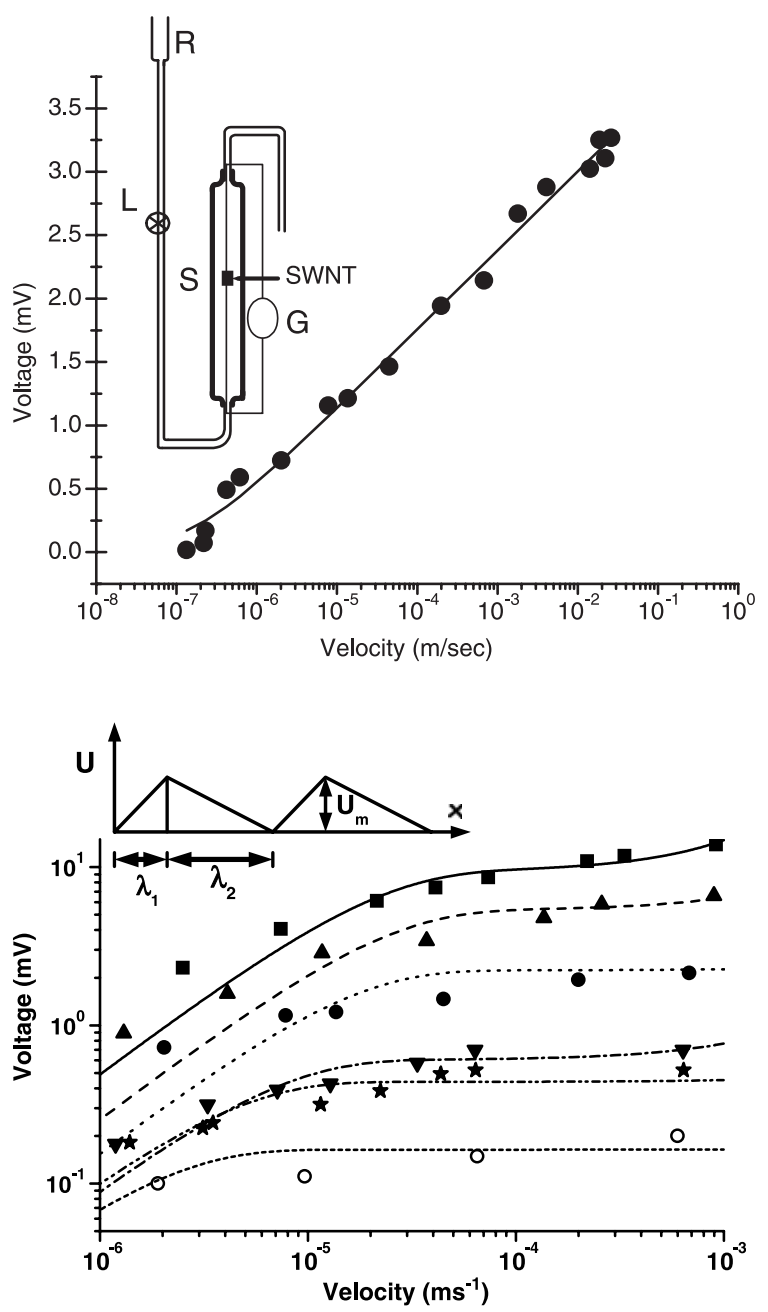

voltage fell with increasing fluid viscosity. We also found that the voltage developed in the case of the polar liquids (such as water) was greater than the voltage developed in the case of the less polar liquids (such as methanol). Thus, for $u=$ $6 \times 10^{-4} \mathrm{~m} / \mathrm{s}, V_{\text {water }}=2.1 \mathrm{mV}$, whereas $V_{\text {methanol }}=0.2 \mathrm{mV}$. Thus, our data set reveals three features: the sublinear (almost logarithmic) voltage response to flow velocity, its strong dependence on the ionic and polar nature of the flowing liquid, and its relatively weak dependence on the liquid viscosity - the latter, in fact, acting to reduce the flow-induced voltage.

The one-dimensional nature of the SWNT is crucial for the generation of a net electrical signal in the sample. Experiments on graphite did not produce any measurable electrical signal. However, experiments with multiwalled carbon nanotubes did generate a voltage, but it was about 10 times smaller than the voltage produced by the SWNT for similar dimensions of the sensing element and for the same flow velocities of the liquid.

The most notable feature of our experimental data is the slow, nearly saturating growth of the induced electric voltage (current) with the increasing flow velocity. This strongly sublinear, almost logarithmic dependence on the flow

Fig. 1. Variation of voltage (V) developed as function of velocity (u) of water. Solid line is a fit to functional form as $V=\alpha \log$ $(u \beta+1)$, where $\alpha$ and $\beta$ are constants. Inset shows experimental setup where $R$ is the reservoir, $L$ is the valve controlling the liquid flow, $\mathrm{S}$ is the cylindrical glass flow chamber, and $G$ is the voltmeter.

Fig. 2. Induced Voltage $(V)$ as function of liquid velocities, for water (0); methanol $(\bigcirc)$, mixture of water:glycerol $(75: 25)$ ( $\star$ ); Water:glycerol $(88: 12)(\boldsymbol{\nabla})$; $0.6 \mathrm{M} \mathrm{HCl}(\boldsymbol{\Delta})$, and $1.2 \mathrm{M} \mathrm{HCl}$ (ם). The ratios for the waterglycerol mixtures are by volume. Lines show the best fit of the data to Eq. 2. Inset shows a schematic of asymmetric ratchet potential. 


\section{R E P O R T S}

velocity rules out any explanation of our experimental results based on a linear response to the fluid flow. We thus suggest a transport mechanism based on the known idea of a directed voltage response resulting from the pulsating asymmetric thermal ratchets $(7,8)$.

An idealized model envisages a one-dimensional periodic ratchet potential that is asymmetric, such as a saw-tooth potential (Fig. 2, inset), and that is modulated in time by a multiplicative process that can be, and usually is, stochastic. Physically, the features that are essential for the rectifying mechanism of this pulsating ratchet to operate are the spatial bias (asymmetry) and the (stochastic) temporal modulation. As for the spatial order, it is physically essential for the sense of the bias to be the same all along the sample. This should enable us to invoke the general Curie principle $(7,9)$ that ensures a directed voltage and current response. These minimal conditions are realized in our case, in which the Coulombic potential resulting from, for example, the fluctuating imbalances in the local charge neutrality of the ionic fluid can provide the stochastic potential, whereas the Poiseuille shear-flow past the nanotube surface provides the unidirectional bias. Thus, one can imagine a transient accumulation of charge imbalance that is shear-deformed by the velocity gradient near the surface, resulting in an asymmetric fluctuating potential. Indeed, a similar idea of shear-induced dipolar (ellipsoidal) distortion of the ion-plus-polar atmosphere at the liquid-solid interface was treated in detail by Onsager and Fuoss (10) in their classic paper on electrolytes (11). Although the exact mapping would involve many details of the fluid-surface interaction, the basic idea is generic in the sense of the Curie principle (9). The one-dimensional confinement of the charge carriers in the nanotube is essential to ensure that the uniformly biased but randomly placed pulsating ratchets along the sample length act additively in series to induce the overall directed voltage. More specifically, the electrostatic ratchet potential would force the nanotube $\pi$-electrons, whose kinetics would resemble that of a classical diffusion in real space, which is consistent with the observed high resistivity of the sample. Again, a symmetrical, fluctuating potential moving with the flow can, in principle, drag charge carriers to induce a voltage. This, however, will give rise to

Table 1. Comparison of the parameters $V_{0}, a$, and $b$ for a series of liquids.

\begin{tabular}{llrr}
\hline \multicolumn{1}{c}{ Liquid } & $\begin{array}{c}V_{0} \\
(\mathrm{mV})\end{array}$ & $a(\mathrm{~s} / \mathrm{m})$ & $b(\mathrm{~s} / \mathrm{m})$ \\
\hline $1.2 \mathrm{M} \mathrm{HCl}$ & 9.2 & $5.4 \times 10^{4}$ & 471 \\
$0.6 \mathrm{M} \mathrm{HCl}$ & 5.3 & $4.8 \times 10^{4}$ & 191 \\
Water & 2.2 & $7 \times 10^{4}$ & 16 \\
Water:glycerol (88:12) & 0.6 & $15 \times 10^{4}$ & 14 \\
Water:glycerol (75:25) & 0.4 & $25 \times 10^{4}$ & 14 \\
Methanol & 0.16 & $53 \times 10^{4}$ & 3 \\
\hline
\end{tabular}

a linear dependence of the induced voltage and current on flow velocity (5), which is in disagreement with our observations.

The inset of Fig. 2 shows a schematic of the idealized asymmetric ratchet potential acting on the charge carriers in the nanotubes. For this idealized model of a pulsating asymmetric ratchet potential, the particle current per particle is known to be given by $(7,12)$

$$
j=\frac{j_{0}\left(1-e^{-\frac{\gamma \lambda^{2} \Delta}{Q U_{m}}}\right)}{\frac{\gamma^{2}}{Q} \int_{0}^{\lambda} \frac{e^{-\varphi(x)}}{\left|U^{\prime}(x)\right|}\left[\int_{x}^{x+\lambda} \frac{\mathrm{e}^{\varphi(y)}}{\left|U^{\prime}(y)\right|} d y d x\right.}
$$

where $\varphi(x)=[(\gamma) /(Q)] \int_{0}^{x}\left\{1 /\left[U^{\prime}(y)\right]\right\} d y$;

prime denotes the derivative with respect to the space coordinate; $\lambda$ is the period (length) of the pulsating ratchet $\left(\lambda=\lambda_{1}+\lambda_{2}\right) ; Q$ is the strength of the fluctuating Coulombic potential assumed to be a white noise; $\gamma$ is the frictional coefficient for the carriers in the nanotubes; $U \mathrm{~m}$ is the maximum of the ratchet potential; and $\Delta=\left[\left(\lambda_{2}-\lambda_{1}\right) /(\lambda)\right]$ is the dimensionless bias (asymmetry) parameter. The above expression for $\varphi(x)$ assumes $\left\{\left[\left(\gamma k \mathrm{~B} T \lambda^{2}\right) /\left(Q U \mathrm{~m}^{2}\right)\right]\right\} \ll 1$, where $k_{\mathrm{B}} T$ is the thermal energy. Here, $\Delta$ is the all-important asymmetry parameter, which should be proportional to the liquid flow velocity. Physically, $\Delta$ can be identified with the dimensionless (dipolar) ellipsoidal deformation of the ionic atmosphere due to the shear-flow at the interface (10) and is on the order of $\left(\Gamma / \kappa^{2}\right.$ $\left.k_{\mathrm{B}} T\right)(u / \delta)$, where $\delta$ is the boundary layer thickness near the nanotube, $\kappa^{-1}$ is the Debye screening length, and $\Gamma$ is the frictional force on an ion moving with unit velocity. Typically, $\delta \sim \sqrt{(v l / u)}$, where $v$ is the kinematic viscosity of the fluid and $l$ is the interface length. For the experimental parameter values, $\Delta \ll 1$.

For small values of $\Delta$ corresponding to small flow velocities, Eq. 1 reduces to give the induced voltage (proportional to the current $j$ ) as

$$
V \simeq \frac{V_{0}\left(1-e^{-a u}\right)}{e^{-b u}}
$$

where $V_{0}, a$, and $b$ are the parameters following from Eq. 1. Figure 2 shows the fit of our data for various liquids to the functional form in Eq. 2 with $V_{0}, a$, and $b$ as the fit parameters (Table 1). The saturating effect is clearly seen and the fit is reasonable over many decades of flow-velocity variation. The addition of $\mathrm{HCl}$ increases the concentration of $\mathrm{H}^{+}$ions (along with their hydration shells), and hence, the observed increase in the induced voltage.

An important point must be made regarding the direction (polarity) of the voltage and current in relation to the fluid flow direction. In our experiments, we observed that the electric current and the fluid were flowing in the same direction. In a linear response theory based on the viscous drag (the phonon wind), the particle current has the same direction as the fluid flow velocity, and, therefore, the sign of the electric voltage and current is determined by the sign of the charge carriers (electron or hole). Thus, for the holelike carriers, the induced voltage and current have the same direction as the flow velocity. This, however, is not the case for a pulsating ratchet model in general (7). For a given direction of the ratchet bias (asymmetry), the direction of the voltage is independent of the sign of the charge on the carriers. It will, of course, depend on the sense of the bias (asymmetry) of the ratchet potential. In a polar fluid of a given ionic strength, such as concentrations of $\mathrm{H}^{+}$and $\mathrm{OH}^{-}$ions (along with their hydration shells), we expect to have ratchet potentials of either sign (bias). The net effect is then determined by the dominant ratchet. What is important here, however, is that there is no a priori (symmetry) reason to expect the oppositely biased ratchets to give an exact cancellation.

Finally, a flow sensor that is based on SWNT directly produces an electrical signal in response to a fluid flow. We believe that this sensor can be scaled down to length dimensions on the order of micrometers - i.e., the length of the individual nanotubes - making it usable in very small liquid volumes. The sensor also has high sensitivity at low velocities and a fast response time (better than $1 \mathrm{~ms}$ ). The nanotubes also could be used to make a voltage and current source in a flowing liquid environment, which may have interesting biomedical applications.

\section{References and Notes}

1. P. G. Collins, M. S. Arnold, P. Avouris, Science 292, 706 (2001)

2. R. H. Baughman et al., Science 284, 1340 (1999).

3. J. Kong et al., Science 287, 622 (2000).

4. R. Egger, A. Gogolin, Phys. Rev. Lett. 79, 5082 (1997)

5. P. Král, M. Shapiro, Phys. Rev. Lett. 86, 131 (2001).

6. P. V. Teredesai et al., Chem. Phys. Lett. 319, 296 (2000).

7. P. Reimann, Phys. Rep. 361, 57 (2002).

8. F. Jülicher, A. Ajdari, J. Prost, Rev. Mod. Phys. 69, 1269 (1997).

9. P. Curie, J. Phys. (Paris) Sér. 3, 3, 393 (1894).

10. L. Onsager, R. M. Fuoss, J. Phys. Chem. 36, 2689 (1932).

11. More microscopically, one may consider the Coulomb potential of an ion undergoing thermal diffusion, fluid advection, and reflection from the fluid-solid interface with aberration caused by the fluid velocity. Such an aberration, much like the shear, provides the unidirectional bias throughout the length of the nanotube.

12. P. Reimann, Phys. Rep. 290, 149 (1997)

13. We thank C. N. R. Rao and A. Govindaraj for SWNT samples and A. Das for his help in performing some of the experiments. A.K.S. thanks the Department of Science and Technology (Government of India) for financial assistance.

4 October 2002; accepted 24 December 2002 Published online 16 January 2003; 10.1126/science. 1079080 Include this information when citing this paper. 